\title{
Status and plans of the Compact Linear Collider Study
}

\author{
Steffen Doebert ${ }^{1}$ \\ CERN \\ Geneva, Switzerland \\ E-mail: steffen.doebert@cern.ch
}

\section{On behalf oft the CLIC collaboration}

The Compact Linear Collider (CLIC) project is exploring the possibility of constructing a multiTeV linear electron-positron collider for high-energy frontier physics studies beyond the LHC era. The CLIC concept is based on high-gradient normal-conducting accelerating structures. The RF power for the acceleration of the colliding beams is produced by a two-beam acceleration scheme, where power is extracted from a high current drive beam that runs parallel with the main linac. The key ongoing studies involve accelerator parameter optimisation, technical studies and component development, alignment and stability, and include a number of system performance studies in test-facilities around the world. The CLIC physics potential and main detector issues, as well as possible implementation staging, are being studied in parallel. A summary of the progress and status of the corresponding studies will be given, as well as an outline of the preparation and work towards developing a CLIC implementation plan by 2018/19.

38th International Conference on High Energy Physics

3-10 August 2016

Chicago, USA 


\section{Introduction}

The Compact Linear Collider study (CLIC) develops a project plan for a staged implementation of an electron-positron collider with a center of mass energy of $3 \mathrm{TeV}$ [1]. CLIC is a two-beam accelerator which takes advantage of high-gradient normal conducting accelerating structures operating at $12 \mathrm{GHz}$. The accelerator is powered by a high-power drive beam generated with high efficiency. Due to the high accelerating gradient of $100 \mathrm{MV} / \mathrm{m}$ a $3 \mathrm{TeV}$ collider could be realized within a length of $50 \mathrm{~km}$. A schematic layout of the $3 \mathrm{TeV}$ machine is shown in figure 1. A central injector complex will house the electron and positron injectors and damping rings as well as the two drive beam accelerators. Drive and main beams are then send to the extremities of the machine for synchronous two beam acceleration. The aim of the CLIC machine is to provide a complementary lepton collider to explore in detail particles and phenomena discovered by the LHC. A staged construction approach is being developed starting with a first stage at $380 \mathrm{GeV}$ to cover higgs and top physics followed by upgrades to $1.5 \mathrm{TeV}$ and finally $3 \mathrm{TeV}$. The higher energy stages should be optimized to study anticipated physics beyond the standard model yet to be discovered by the LHC. The CLIC study at CERN is mandated to provide a project plan as input to the next European strategy update on high energy physics scheduled for 2019. Construction of CLIC could start around 2025 in case of a positive decision.

While the CDR in 2012 was centered around the feasibility of the $3 \mathrm{TeV}$ machine, in the current development phase we focus on the parameter optimization of the first $380 \mathrm{GeV}$ stage and the subsequent staging strategy. Performance verification using several test facilities around the world (CTF3, FACET, ATF) is ongoing as well as key-hardware developments. An emphasis has been put on reduction of cost and power consumption. In addition the CLIC study actively promotes the use of CLIC technologies for other application such as FEL's and medical accelerators.

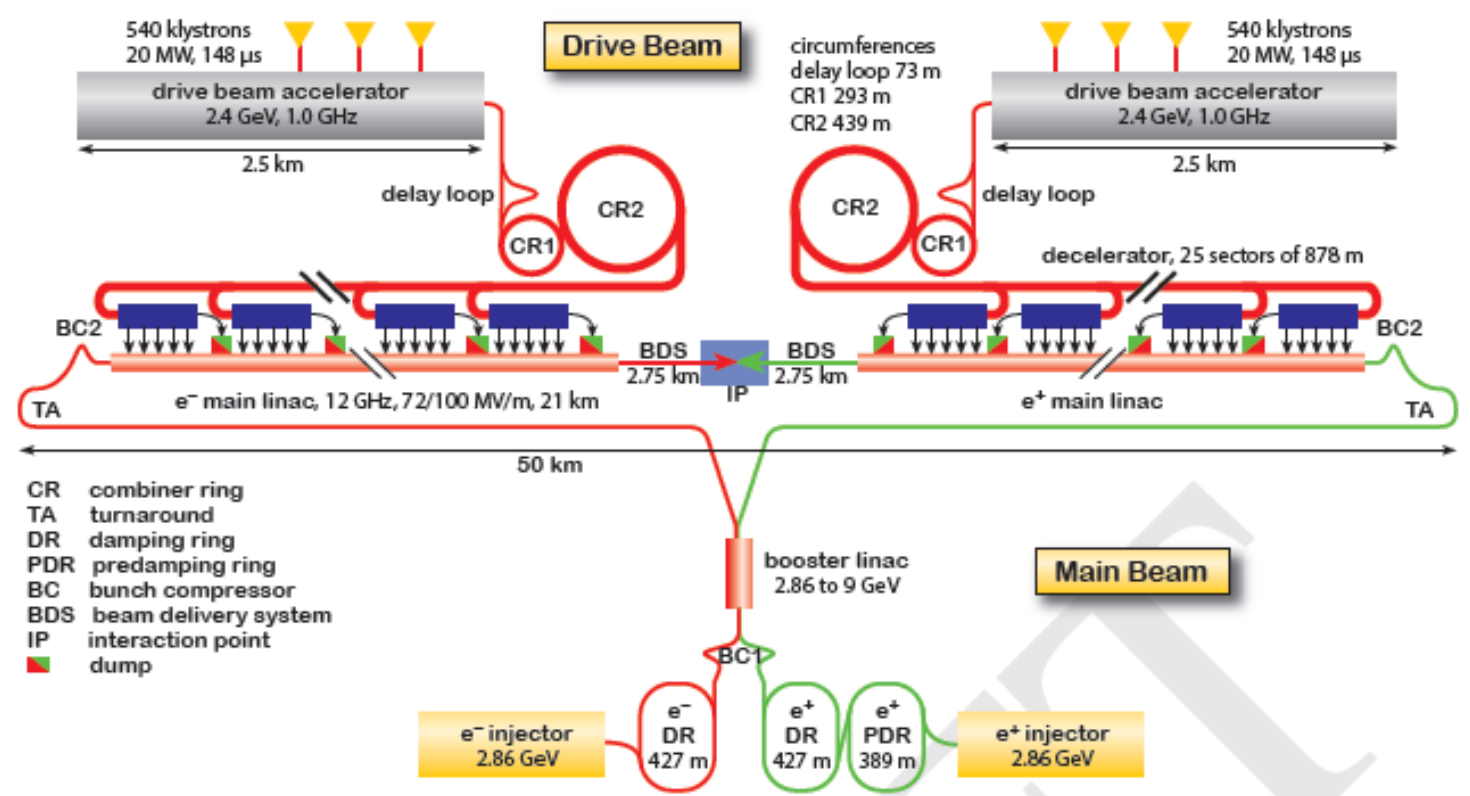

Figure 1: Schematic layout of CLIC accelerator complex with a center of mass energy of 3 $\mathrm{TeV}$ 


\section{First stage of CLIC at $380 \mathrm{GeV}$}

The CLIC physics and detector collaboration studied the physics case for different staging scenarios and recommended at first stage at $380 \mathrm{GeV}$ and subsequent stages at 1.5 and 3 $\mathrm{TeV}$ [2]. The CLIC design has a complex interplay of its beam parameters in the different parts of the machine therefore a sophisticated model has been developed to optimize these parameters. The target luminosity is used as a global input parameter, then many different machine designs are created and their cost and power consumption are evaluated. Central to the approach are the parameters of the accelerating structure which then determines a large part of the design. A few million variants have been evaluated efficiently in this process revealing the most promising candidates. It turned out that the machines with the lowest power consumption have as well the lowest cost. Luminosity can be added by increasing cost and power consumption. The optimum found, uses an accelerating gradient of $72 \mathrm{MV} / \mathrm{m}$ for the first stage and slightly longer bunch trains and higher bunch charge. The overall length of such a $380 \mathrm{GeV}$ machine would be $11.4 \mathrm{~km}$ with a power consumption of $252 \mathrm{MW}$ for a luminosity of $1.510^{34} \mathrm{~cm}^{-2} \mathrm{~s}^{-1}$.

This choice of parameters has the advantage that the machine could be upgraded later on with $100 \mathrm{MV} / \mathrm{m}$ structure up to $3 \mathrm{TeV}$ in a relatively straight forward way since the drive beam parameters are very similar. Table 1 shows the main parameters for the three possible stages of CLIC in such a scenario. The $380 \mathrm{GeV}$ stage would be powered by a single drive beam with a shorter pulse length. For an upgrade to higher energies the 72 $\mathrm{MV} / \mathrm{m}$ modules would be moved to the beginning of the linac and the additional space would be filled with $3 \mathrm{TeV}$ type $100 \mathrm{MV} / \mathrm{m}$ modules. For the final stage at $3 \mathrm{TeV}$ a second drive beam needs to be added.

\begin{tabular}{llllll}
\hline Parameter & Symbol & Unit & Stage 1 & Stage 2 & Stage 3 \\
\hline Centre-of-mass energy & $\sqrt{s}$ & $\mathrm{GeV}$ & 380 & 1500 & 3000 \\
Repetition frequency & $f_{\text {rep }}$ & $\mathrm{Hz}$ & 50 & 50 & 50 \\
Number of bunches per train & $n_{b}$ & & 352 & 312 & 312 \\
Bunch separation & $\Delta t$ & $\mathrm{~ns}$ & 0.5 & 0.5 & 0.5 \\
Pulse length & $\tau_{\mathrm{RF}}$ & $\mathrm{ns}$ & 244 & 244 & 244 \\
\hline Accelerating gradient & $G$ & $\mathrm{MV} / \mathrm{m}$ & 72 & $72 / 100$ & $72 / 100$ \\
\hline Total luminosity & $\mathscr{L}$ & $10^{34} \mathrm{~cm}^{-2} \mathrm{~s}^{-1}$ & 1.5 & 3.7 & 5.9 \\
Luminosity above 99\% of $\sqrt{s}$ & $\mathscr{L}_{0.01}$ & $10^{34} \mathrm{~cm}^{-2} \mathrm{~s}^{-1}$ & 0.9 & 1.4 & 2 \\
\hline Main tunnel length & & $\mathrm{km}$ & 11.4 & 29.0 & 50.1 \\
Number of particles per bunch & $N$ & $10^{9}$ & 5.2 & 3.7 & 3.7 \\
Bunch length & $\sigma_{z}$ & $\mu \mathrm{m}$ & 70 & 44 & 44 \\
IP beam size & $\sigma_{x} / \sigma_{y}$ & $\mathrm{~nm}$ & $149 / 2.9$ & $\sim 60 / 1.5$ & $\sim 40 / 1$ \\
Normalised emittance (end of linac) & $\varepsilon_{x} / \varepsilon_{y}$ & $\mathrm{~nm}$ & $920 / 20$ & $660 / 20$ & $660 / 20$ \\
Normalised emittance (at IP) & $\varepsilon_{x} / \varepsilon_{y}$ & $\mathrm{~nm}$ & $950 / 30$ & - & - \\
Estimated power consumption & $P_{\text {wall }}$ & $\mathrm{MW}$ & 252 & 364 & 589 \\
\hline
\end{tabular}

Table 1: CLIC parameters for the three stages of the machine.

3. Recent development highlights 
The CLIC study focused in the last years on the development of key-technologies which have a significant impact on cost, power consumption, schedule or performance. Many key studies are ongoing from which only a few can be mentioned in this summary.

To reduce the power consumption permanent magnet solutions are actively studied. A good example are the quadrupoles needed for the decelerator part of the drive beam. More than 20000 magnets are needed with an integrated gradient as high as $14 \mathrm{~T}$ at the beginning of the decelerator down to about $1 \mathrm{~T}$ at the end. Two tuneable permanentmagnet quadrupoles have been developed by STFC able to cover the whole dynamic range required. The concept is based on fixed pole pieces and moveable permanent magnets increasing the field in the pole pieces. Measurements on prototypes demonstrated that the field quality can be achieved and the magnets reliably tuned [3]. Figure 2 show a picture of such a prototype. Using this option for CLIC would save about 30 MW of power.

The development of very high efficiency rf power sources is another example for the effort to reduce power consumption for the machine. The power to the CLIC beams would be mainly supplied by 1080 such klystrons via the drive beams. Together with industry, prototypes of the drive beam klystron with a target efficiency of $70 \%$ have been launched. Recently the first prototype successfully finished its factory acceptance test with a measured efficiency of $72 \%$ presenting a major milestone for the drive beam R\&D.

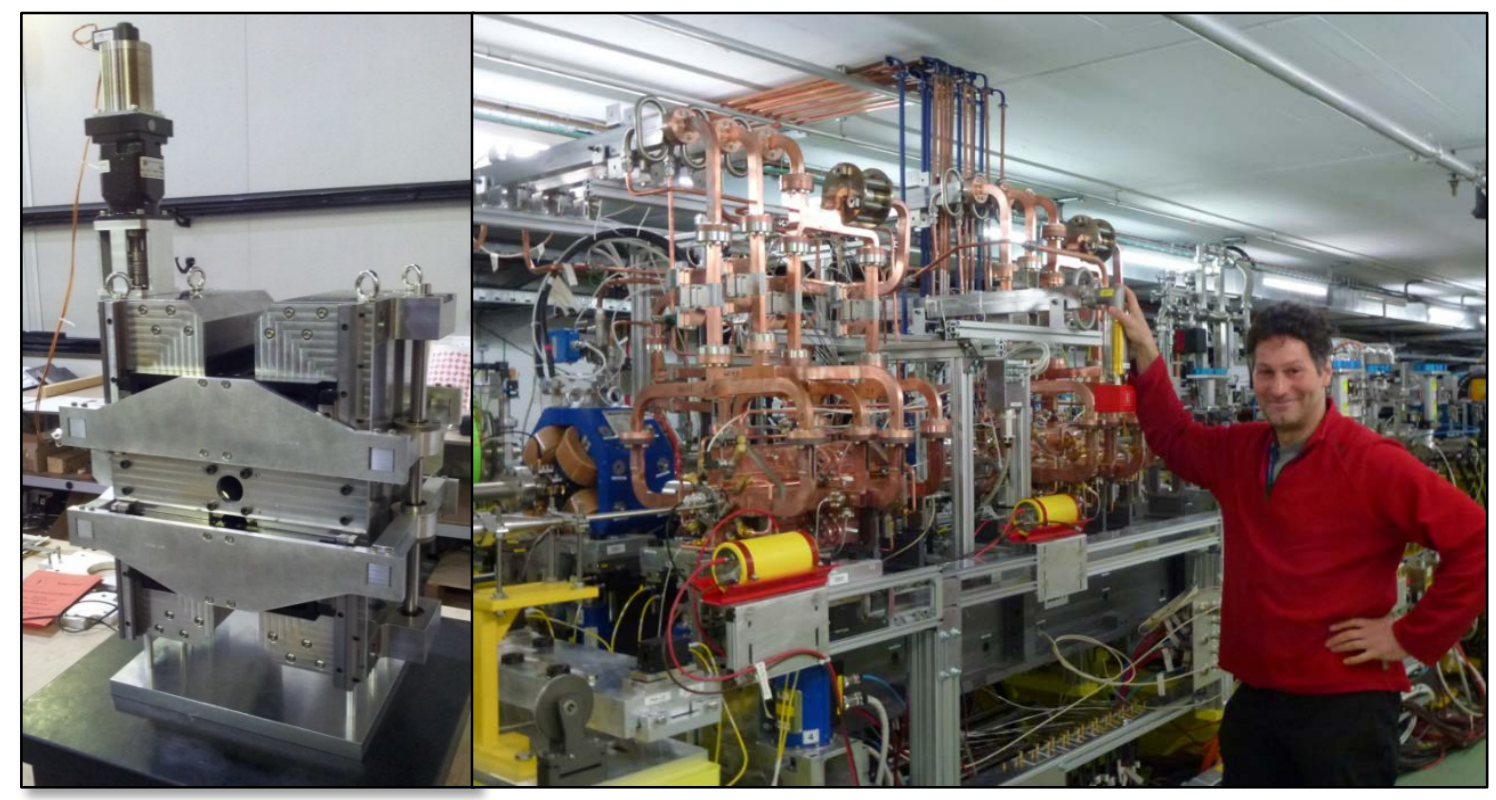

Figure 2: Left: Tuneable permanent magnet quadrupole prototype for the drive beam decelerator. Right: Fully equipped two beam module installed in the the CLIC test facility (CTF3) at CERN.

Concerning performance verification and optimization many experiments are carried out using accelerator test facilities around the world. In ATF at KEK (Japan) the final focus system for ILC and CLIC is studied in a common effort with major contributions of hardware and manpower from the CLIC team. In FACET at SLAC (US) dispersion free steering has been demonstrated with great success showing the feasibility of the CLIC emittance preservation strategies [4]. Finally in CTF3 at CERN substantial progress has been made to validate the phase feed-forward system of the drive beam to correct beam phase errors around the complex [5]. An arrival phase stability of 0.3 deg of $12 \mathrm{GHz}$ has 
been achieved. The test beam line for the decelerator obtained more than $50 \%$ energy extraction producing $1.3 \mathrm{GW}$ of $12 \mathrm{GHz}$ rf peak power. Finally a fully equipped prototype CLIC two beam module is being used in CTF3. A highlight of the experiments around this module was the measurement of $5 \mu \mathrm{m}$ resolution for the wake field monitors integrated into the accelerating structures [6]. These monitors allow the precise beam based alignment in CLIC to mitigate the wake field effects. The $3 \mathrm{TeV}$ machine consists of about 20000 such two beam modules. A dedicated program studies the alignment, mechanical integration and industrialization

The x-band testing capabilities at CERN have been extended to three klystron based test stands. One test stand is used together with the beam of CTF3 to investigate the effect of beam loading on $\mathrm{rf}$ breakdowns. So far the measurements indicate that the breakdown rate is dominated by the maximum surface field along the structure rather than the detailed beam loading profile [7]. There is an increasing interest in the CLIC x-band technologies for applications like a compact FEL, compact booster linacs for light sources and medical applications driven by the desire of reduction of cost and space requirements. The CLIC study is actively promoting these applications of linear collider technologies.

\section{Conclusions}

The CLIC study has a well-defined R\&D program for the project preparation phase leading up to the next European strategy update for high energy physics in 2019. The collaboration comprises currently 50 institutes in more than 30 countries.

The focus is on developing a credible staged approach to construct a $3 \mathrm{TeV}$ linear collider with emphasis on the first stage at $380 \mathrm{GeV}$. New findings at the LHC will have to be incorporated into the project plane. Performance verification efforts and the development of key technologies are ongoing. In particular we aim to reduce the project cost and power consumption without compromising the performance of the machine.

\section{References}

[1] “A Multi-TeV Linear Collider base on CLIC Technology: CLIC Conceptual Design Report”, edited by M. Aichler, P. Burrows, M. Draper, T. Garvey, P. Lebrun, K. Peach, N. Phinney, H. Schmickler, D. Schulte, N. Toge, CERN-2012-007 (CERN, Geneva, 2012)

[2] “Updated baseline for a staged compact linear collider”, edited by P.N. Burrows, P. Lebrun, L. Linsen, D. Schulte, E. Sicking, S. Stapnes, M.A. Thomson, CERN-2016-xxxx (CERN, Geneva, 2016)

[3] P. Wadhwa et al., "Design and Measurement of a Low-energy Tuneable Permanent Magnet Quadrupole Prototype", ’PAC 2014, Dresden, Germany, 2014

[4] A. Latina et al., "Experimental demonstration of a global dispersion-free steering correction at the new linac test facility at SLAC”, Phys. Rev. ST Accel. Beams 17, 042803, 2014

[5] J. Roberts et al. , "Demonstration of CLIC Level Phase Stability Using a High Bandwidth, Low Latency, Drive Beam Phase Feedforward System at the CLIC Test Facility CTF3”, IPAC 2016, Busan, South Korea, 2016.

[6] R.L. Lillestol et al., "Status of Wakefield Monitor Experiments at the CLIC test facility”, IPAC 2016, Busan, South Korea , 2016

[7] F. Tecker et al., "Beam-Loading effect on breakdown rate in high-gradient accelerating structures”, IPAC 2016, Busan, South Korea , 2016 